\title{
The wisdom of the body; a contemporary view
}

\section{Bruce J.West*}

Physics Department, Duke University, Durham, NC, USA

*Correspondence: bwest@nc.rr.com

I know that most men, including those at ease with the problems of the greatest complexity, can seldom accept even the simplest and most obvious truth if it be such as would oblige them to admit the falsity of conclusions which they have delighted in explaining to colleagues, which they have proudly taught to others, and which they have woven, thread by thread, into the fabric of their lives.

L. Tolstoy

Fractal Physiology (Bassingthwaighte et al., 1994) opens with the above quotation denoting a conscious break with the traditional methods of researching the fundamental questions in human physiology as did a somewhat more modest attempt a few years earlier (West, 1990). By and large there is a single guiding principle explaining how the human body operates independently of whether an investigator is a clinician, researcher or a combination of the two, and that principle is homeostasis. The human body has multiple automatic inhibition (i.e., negative feedback) mechanisms that suppress disquieting influences, some of which can be controlled and other which cannot. Homeostasis is the strategy, the culmination of countless years of evolution, by which the body reacts to changes in environmental stimuli with equilibrating responses (Cannon, 1932). This equilibrium perspective of physiology co-evolved with the mathematical/engineering notion of control theory that was so successful in the design engineering of the products and systems of the industrial age and subsequently the information age. However, just as traditional control theory is not adequate for designing truly complex adaptive systems or devices, so too homeostasis lacks the dynamic flexibility to describe the richness of the non-equilibrium phenomena in physiology (West, 2006). Frontiers in Fractal Physiology is intended to attract research papers concerning how to modernize, update and where necessary step beyond homeostasis to understand physiological phenomena.

Nearly two decades ago fractal physiology was identified as an outgrowth of the pioneering research into the applications to biomedicine of a number of the then newly formed mathematical ideas by a handful of investigators studying the developing areas of nonlinear dynamics and complex adaptive systems. One fundamental issue concerned measurement and how the notion of a characteristic scale determines what we think we know about the quantities we measure. Mandelbrot (1977) brought to the world's attention the fact that many natural objects simply do not satisfy this preconception; what is measured is not independent of how the measurement is done. Living things have structures in space and fluctuations in time that cannot be characterized by one spatial or temporal scale, but extend over many scales. Mandelbrot introduced the notion of fractal geometry and fractal statistics to describe this lack of a characteristic scale in both physiologic structure and dynamics. If we magnify (use a smaller ruler) a fractal structure, new and ever finer details are revealed, whether the structure is in space or time. Consequently, a cognizance of fractals and its mathematical elaborations provides the tools needed to describe, measure, model, and understand many objects and processes in living things.

One of the Grand Challenges of Fractal Physiology is to identify the foundational principles that entail the functional and structural organization of the human body. In particular how does identifying and interpreting fractality by means of scaling of complex physiologic phenomena necessitate dynamic principles with which to replace and/or compliment homeostasis? More generally, how are the structure, function and activity of organs, tissue and cells revealed in the coupling across space/time scales as well as in the transport of information within and between functional and regulatory networks?

However, the fractal idea was only one of many mathematical concepts woven into the initial fabric of Fractal Physiology. Another useful idea was that of self-similarity; a concept related to the structure within structure motif of fractals and which is manifest in time series data through scaling indices (West and Grigolini, 2010). The scaling index is a measure of the variability in the cardiovascular system, the respiratory system, and the motor control system among others, and suggests new ways to characterize physiologic phenomena based on variability rather than traditional average values. The scaling index quantifies how the multiple scales contributing to a complex process are coupled together and suggests new mathematical models for the underlying dynamics. For example, when the scaling index changes over time the underlying process is multi-fractal and the coupling across scales can be non-stationary and consequently non-ergodic.

Of course mathematical models (theory) are only useful in science if they are able to provide a coherent interpretation of the patterns (information) observed in data. Consequently, this journal not only welcomes papers on mathematical theory of complex physiologic phenomena but in addition solicits manuscripts on the processing of both experimental and observational data sets that test these various models. Submissions are encouraged that address questions concerning how an individual system scales; how the scaling in one physiologic system influences that of another and what such influence entails about structure and function.

\section{REFERENCES}

Bassingthwaighte, J. B., Liebovitch, L. S., and West, B. J. (1994). Fractal Physiology. New York: Oxford University Press.

Cannon, W. B. (1932). The Wisdom of the Body. NY: W. W. Norton. 
Mandelbrot, B. B. (1977). Fractals, Form and Chance. San Francisco: W. H. Freeman.

West, B. J. (1990). Fractal Physiology and Chaos in Medicine, Studies of Nonlinear Phenomena in Life Science-Vol 1. Teaneck, NJ: World Scientific.

West,B.J.(2006). Where Medicine Went Wrong: Rediscovering the Path to Complexity, Studies of Nonlinear Phenomena in Life Science-Vol 11. Teaneck, NJ: World Scientific.
West,B.J.and Grigolini,P.(2010).ComplexWebs:Anticipating the Improbable. Oxford: Camridge University Press.

Received: 16 March 2010; accepted: 17 March 2010; published online: 23 April 2010.

Citation: West BJ (2010) The wisdom of the body; a contemporary view. Front. Physio. 1:1. doi: 10.3389/ fphys.2010.00001
This article was submitted to Frontiers in Fractal Physiology, a specialty of Frontiers in Physiology.

Copyright (c) 2010 West. This is an open-access article subject to an exclusive license agreement between the authors and the Frontiers Research Foundation, which permits unrestricted use, distribution, and reproduction in any medium, provided the original authors and source are credited. 